\title{
OPEN Rhizobia use a pathogenic-like effector to hijack leguminous nodulation signalling
}

\author{
Safirah Tasa Nerves Ratu ${ }^{1}$, Albin Teulet ${ }^{2}$, Hiroki Miwa ${ }^{3}$, Sachiko Masuda ${ }^{3}$, Hien P. Nguyen ${ }^{1}$, \\ Michiko Yasuda ${ }^{3}$, Shusei Sato ${ }^{4}$, Takakazu Kaneko ${ }^{5}$, Makoto Hayashi ${ }^{6}$, Eric Giraud ${ }^{2}$ \& \\ Shin Okazaki ${ }^{1,3 \otimes}$
}

Legume plants form a root-nodule symbiosis with rhizobia. This symbiosis establishment generally relies on rhizobium-produced Nod factors (NFs) and their perception by leguminous receptors (NFRs) that trigger nodulation. However, certain rhizobia hijack leguminous nodulation signalling via their type III secretion system, which functions in pathogenic bacteria to deliver effector proteins into host cells. Here, we report that rhizobia use pathogenic-like effectors to hijack legume nodulation signalling. The rhizobial effector Bel2-5 resembles the XopD effector of the plant pathogen Xanthomonas campestris and could induce nitrogen-fixing nodules on soybean $n f r$ mutant. The soybean root transcriptome revealed that Bel2-5 induces expression of cytokinin-related genes, which are important for nodule organogenesis and represses ethylene- and defense-related genes that are deleterious to nodulation. Remarkably, Bel2-5 introduction into a strain unable to nodulate soybean mutant affected in NF perception conferred nodulation ability. Our findings show that rhizobia employ and have customized pathogenic effectors to promote leguminous nodulation signalling.

Symbiotic interactions between legume plants and soil bacteria, collectively called rhizobia, result in the formation of nitrogen-fixing root nodules on host plant roots. In nodules, rhizobia fix atmospheric dinitrogen as ammonia to provide host plants with nitrogen nutrition. In exchange, the host plants provide steady carbon sources and essential nutrients as well as an appropriate environment required for bacterial metabolism and development ${ }^{1}$. Due to this symbiosis, legumes play a critical role in agriculture and global nitrogen cycles ${ }^{2,3}$.

Root-nodule symbiosis involves highly host-specific interactions-a rhizobium species can nodulate only limited legume-plant species-and this specificity is determined by several layers of signal exchange between rhizobia and host plants ${ }^{4}$. First, plants secrete flavonoids from roots that are specific to plant species; their recognition by the transcriptional regulator NodD activates the expression of nodulation (nod) genes, leading to the synthesis of rhizobial signal molecules, named Nod factors (NFs), which are lipochitooligosaccharides harbouring species-specific modifications. NF recognition by plant LysM receptors at the plasma membrane activates symbiotic signalling in the host, leading to nodule formation and bacterial infection ${ }^{5,6}$. Second, bacterial extracellular polysaccharides, such as exopolysaccharides (EPSs), lipopolysaccharides (LPSs), and capsular polysaccharides (KPSs), function as another layer of host-specificity determinants ${ }^{7-9}$.

A third specific layer was shown to be constituted by rhizobial effector proteins secreted by type III/IV secretion systems (T3SS/T4SS), providing cultivar-specific and strain-specific incompatibility between two partners ${ }^{10}$. T3SS/T4SS were originally identified in pathogenic bacteria, and the secreted proteins (T3SS effectors; T3Es) were reported to function in bacterial infection and pathogenicity. Many rhizobia also possess functional T3SS/T4SS that secrete various effectors upon induction by host flavonoids ${ }^{11}$. Depending on the host genotype, rhizobial

${ }^{1}$ United Graduate School of Agricultural Science, Tokyo University of Agriculture and Technology, Saiwaicho 3-5-8, Fuchu, Tokyo 183-8509, Japan. '2Laboratoire Des Symbioses Tropicales Et Méditerranéennes, Institut de Recherche Pour Le Développement, UMR Institut de Recherche Pour Le Développement/SupAgro/Institut National de Recherche Pour L'Agriculture, L'Alimentation Et L'Environnement, Université de Montpellier/Centre de Coopération Internationale en Recherche Agronomique Pour Le Développement, 34398 Montpellier Cedex 5, France. ${ }^{3}$ Department of International Environmental and Agricultural Science, Graduate School of Agriculture, Tokyo University of Agriculture and Technology, Saiwaicho 3-5-8, Fuchu, Tokyo 183-8509, Japan. ${ }^{4}$ Graduate School of Life Sciences, Tohoku University, Katahira, Aoba-ku, Sendai, Miyagi 980-8577, Japan. ${ }^{5}$ Faculty of Life Sciences, Kyoto Sangyo University, Motoyama, Kamigamo, Kita-Ku 603-8555, Japan. ${ }^{6}$ Center for Sustainable Resource Science, RIKEN, 1-7-22 Suehiro-cho, Tsurumi-ku, Yokohama City, Kanagawa 230-0045, Japan. ${ }^{\boxplus}$ email: sokazaki@ cc.tuat.ac.jp 
effectors can favour or impede root nodule formation. For example, in Ensifer fredii (S. fredii) NGR234, NopM acts as an E3 ubiquitin ligase and promotes nodulation by potentially reducing reactive oxygen species (ROS) generation in host plants during infection, whereas NopL can serve as a mitogen-activated protein kinase (MAPK) substrate and interfere with MAPK signalling to prevent premature nodule senescence ${ }^{12,13}$. In contrast, two effectors of Bradyrhizobium elkanii USDA61, InnB and Bel2-5, negatively affect nodule formation in some legume plants $^{14,15}$. The negative effects of effectors frequently result from the induction of the host defence response called effector-triggered immunity (ETI), which occurs after the perception of effectors by plant resistance [R] proteins. ETI is often associated with a hypersensitivity response that halts rhizobial infection. These few examples illustrate that a root-nodule symbiosis requires evasion or suppression of the host immune responses, and thus, the strategy of legume infection by rhizobia displays some similarity with those used by plant pathogens ${ }^{16}$.

In addition to modulating plant immunity, the T3SS in some Bradyrhizobium strains was shown to activate nodulation processes in some legume species in the absence of NF signalling ${ }^{17-19}$. This effect was observed for Bradyrhizobium sp. ORS3257, which induces nodule formation on Aeschynomene indica not by NF signalling but via a cocktail of effectors that play synergistic and complementary roles. Remarkably, it was shown that ectopic expression of one of these effectors (ErnA) in transgenic A. indica lines activates organogenesis of root and nodule-like structures ${ }^{19}$. A similar scenario was also described for nodulation of a soybean $n f r 1$ mutant that can be induced by a USDA61 mutant unable to synthesize NFs, but that has a functional T3SS, suggesting that T3Es can bypass NF perception. One possibility would be that an as-yet-unidentified T3 effector activates NF signalling and induces the expression of symbiotic genes, such as ENOD40 and NIN ${ }^{17}$. In these two cases, root hair curling and infection threads, two typical NF-induced responses, were not observed, which suggests that some T3Es can promote intercellular infection ${ }^{17,18}$.

To advance our understanding of this T3SS hijacks NF signalling, we identified the effectors governing nodule organogenesis during the symbiosis between USDA61 and soybean plants. We found that the effector Bel2-5, which resembles XopD, a T3E of plant pathogenic Xanthomonas bacteria, is a key factor of this alternative symbiotic process. The effector targets the plant nucleus and encodes an ubiquitin-like protease 1 (ULP1) domain which is required for triggering nodulation. More interestingly, Bel2-5 not only dampens several plant defencerelated responses, as a phytopathogenic effector, but also plays a distinct role in the induction of a complete set of cytokinin biosynthesis-related genes essential for nodule organogenesis.

\section{Results}

Screening of T3E candidates trigger nodulation on nfr1 mutant soybean. Previously, we reported that $B$. elkanii USDA61 harbours a unique set of T3Es that can hijack soybean NF signalling to promote infection ${ }^{17}$. We hypothesized that specific T3E(s) activate a series of symbiosis signalling steps, leading to efficient nodulation on $n f r 1$ mutant soybean. It was previously shown that the ErnA effector of the USDA61 strain plays a key role during symbiosis with $A$. indica and that the ernA mutant displays an apparent nod minus phenotype in this legume species ${ }^{19}$. We first analysed the effect of the ernA mutant, designated as BEernA, on En1282 (nfr1-mutant soybean). As shown in Fig. 1a, the nodulation properties of the ernA mutant on En1282 plants were significantly affected, but nodules were still formed at $\sim 60 \%$ of the rate for the wild-type (WT) strain, indicating that other effector(s) could play a more prominent role in nodulation.

To identify the responsible effector, we generated mutants of USDA61 in putative effectors previously recognized based on two in silico searches, (1) genome TBLASTN searches for effector homologues in reference to identified rhizobial T3Es and (2) $t$ ts box searches for unidentified effectors putatively regulated by $t t s I$, a specific modulator of rhizobial T3SSs ${ }^{20,21}$, using the USDA61 genome (accession nos. AP013103 and AP013104). By combining these approaches, 54 putative effectors were predicted (Supplementary Table 1). Considering the large number of effector candidates, we decided to focus our mutagenesis approach on (1) the homologues of effector genes that were previously reported to have symbiotic roles in other rhizobia (nopL, nopM, and nopP) and (ii) USDA61-specific putative effector genes (orf48 and orf 91$)^{22}$. Thus, we mutated 7 putative effectors and designated the mutants BEnopL, BEnopM1, BEnopM2, BEnopP1, BEnopP2, BEorf48, and BEorf91. As certain rhizobial effectors can affect symbiosis positively or negatively with different host plants ${ }^{23-25}$, we also included in this analysis two previously obtained mutants in T3Es shown to negatively impact the symbiotic interaction with specific legumes: (1) a deletion mutant of InnB, which was shown to be responsible of the incompatibility of USDA61 with Vigna radiata KPS1 plants ${ }^{14}$, and (2) a transposon mutant in the putative effector Bel2-5, named BE2-5; the Bel2-5 effector was shown to be responsible for the incompatibility of USDA61 with Rj4 soybean plants ${ }^{15}$.

On these additional 9 mutants tested, BEnopL and BE2-5 displayed symbiotic phenotypes (Fig. 1a). The nopL mutant induced $\sim 20 \%$ less nodule formation than the WT strain, while the Tn 5 bel2-5 mutant displayed a strict nod minus phenotype, as observed for a mutant in the T3SS machinery (BErhcJ) (Fig. 1a). To confirm that the phenotype of BE2-5 was not due to a polar effect of the Tn5 insertion, we constructed a deletion mutant of this gene and complemented it with the bel2-5 gene, designated $\Delta$ bel2-5 and $\Delta$ bel2-5::bel2-5, respectively. The bel2-5 deletion abolished En1282 nodulation, and furthermore, the nodulation phenotype was restored in plants inoculated with the complemented bel2-5 mutant strain, resembling that observed with WT USDA61 (Fig. 1b,c). Together, these results demonstrated that Bel2-5 plays a prominent role to promotes nodulation on $n f r 1$ soybean, while the ErnA and NopL effectors appear to be less essential during symbiosis with this host plant.

Bel2-5 is conserved among T3SS-harbouring rhizobia and plant pathogenic bacteria. Bel2-5 was predicted to possess a T3SS-related function, considering the presence of a $t$ ts box in its promoter region (Supplementary Data 1a). Furthermore, the first 50 aa residues of Bel2-5 feature a high proportion of Ser and a low proportion of Leu and Lys, and the fourth residue in Bel2-5 is also preceded by a Pro (Supplementary Data $1 \mathrm{~b})$, thus satisfying the criteria for a T3E $\mathrm{E}^{26,27}$. BLASTP searches revealed that Bel2-5 is distributed among rhizo- 


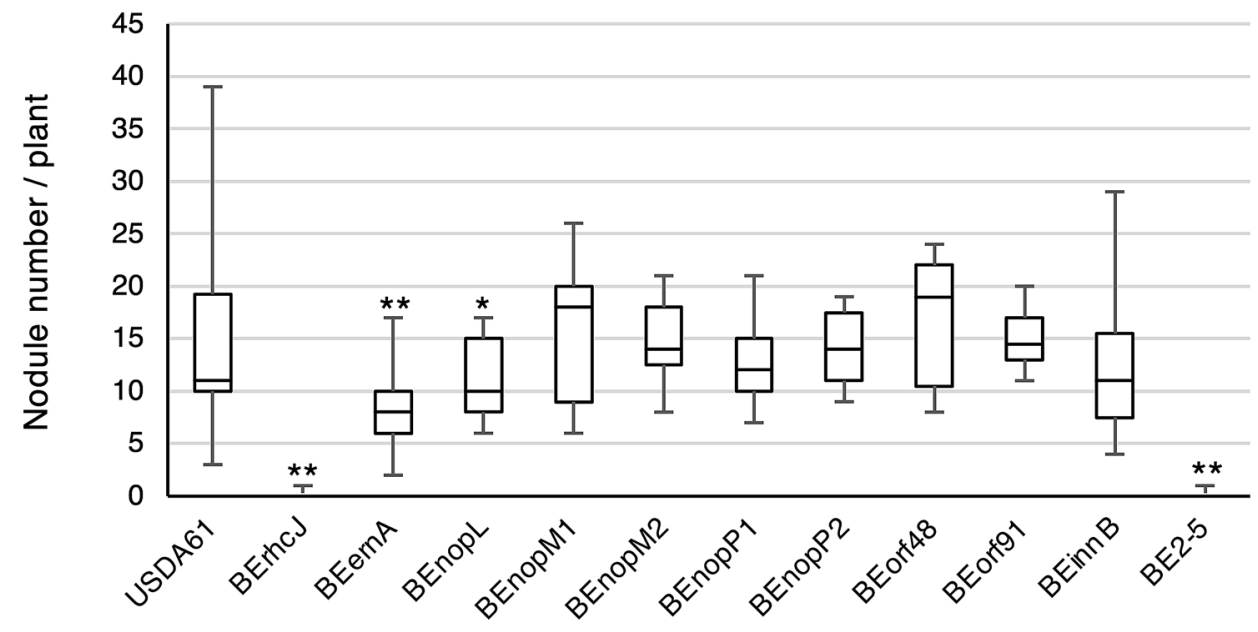

b

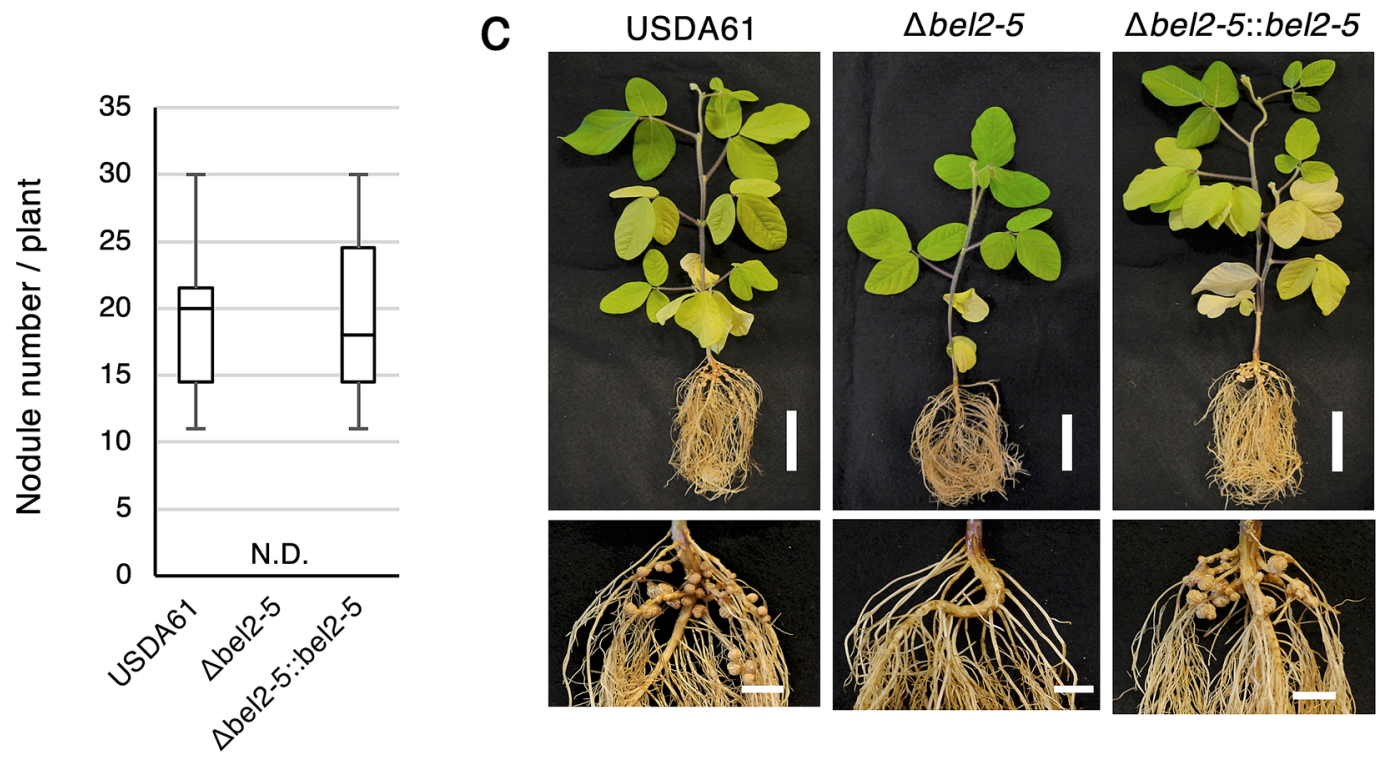

Figure 1. Nodulation of G. $\max \mathrm{cv}$. En1282 (nfr1 mutant soybean) inoculated with wild-type and nops mutant strains of B. elkanii USDA61. (a) The number of nodules formed on En1282 inoculated with USDA61 wildtype and nops mutant candidates at 30 days after inoculation (dai). Values represent mean $\pm \mathrm{SD}(n=36$ for USDA61, from four independent inoculation tests; $n=27$ for BErhCJ; $n=29$ for BEernA; $n=25$ for BEnopL and BEnopM1; $n=23$ for BEnopM2; $n=21$ for BEnopP1; $n=19$ for BEnopP2 and BEinnB; $n=20$ for BEorf48, $n=22$ for BEorf91, and $n=24$ for BE2-5; from three independent inoculation tests for all mutants). (b) The number of nodules formed on En1282 inoculated with bel2-5 mutant of B. elkanii strains at 30 dai. Values represent mean $\pm \mathrm{SD}$ ( $n=15$, from three independent inoculation tests). N.D. means not detected. (c) En1282 plants inoculated with B. elkanii and bel2-5 mutant strains. Plants were photographed at 30 dai. Scale bars: $5 \mathrm{~cm}$ for whole plants and $1 \mathrm{~cm}$ for roots. ${ }^{\star} p<0.05$ and ${ }^{* *} p<0.001$ by two-tailed Student $t$-test compared to USDA61 wild-type (a,b).

bia, including Bradyrhizobium, Sinorhizobium (Ensifer), and Mesorhizobium species. However, Bel2-5 seems not to be a rhizobium-specific effector since it shares some similarities with XopD and XopD-like PsvA effectors from the phytopathogenic bacteria Xanthomonas campestris pv. vesicatoria (Xcv.) str. 85-10 (DAA34040.1; 26.11\% identity) and Pseudomonas syringae pv. eriobotryae (BAA87062.1; 25.60\% identity) in the C-terminal region of the two proteins, respectively (Fig. 2).

A putative nuclear localization signal (NLS; RPAKRPRTL) was detected at 788-796 aa in Bel2-5 using NLS mapper $^{28}$ (Fig. 2b,c; Supplementary Data 1b), which indicates potential nuclear translocation. Interestingly, ErnA and XopD also contain putative NLSs, and their localization to the plant nucleus has been demonstrated ${ }^{19,29}$. Two sets of tandem repeat sequences with unknown function were found at 148 to 438 aa (containing two repeats of 146 residues) and at 932 to 1109 aa (containing five repeats, with four first repeats consist of 36 residues, and last repeat consists of 33 residues) of Bel2-5 (Fig. 2b,c; Supplementary Data 1b). Sequence analysis study with 
a
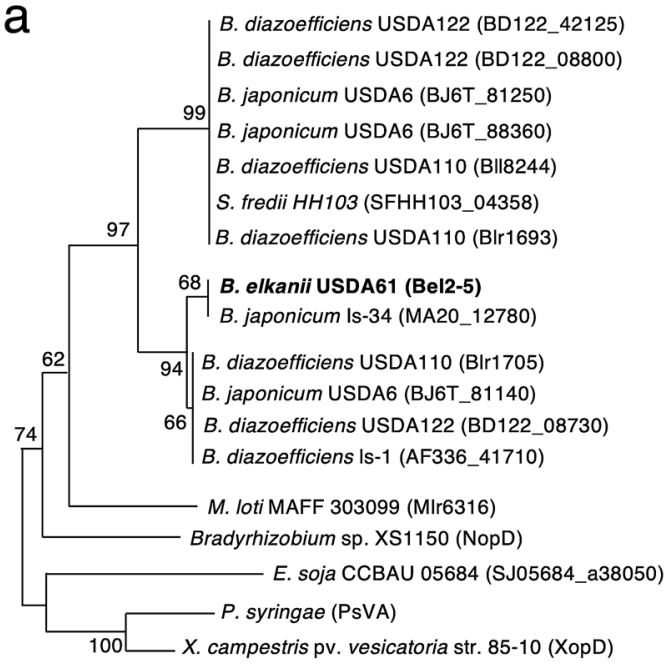

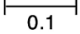

b

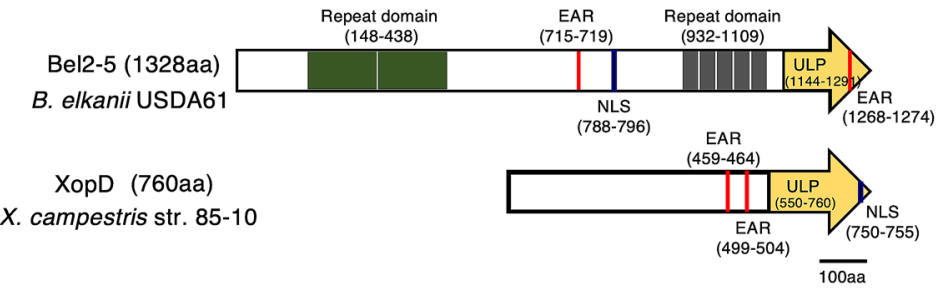

C

USDA61

Is-34

USDA110

S. fredii $\mathrm{HH} 103$

XS1150 (NopD)

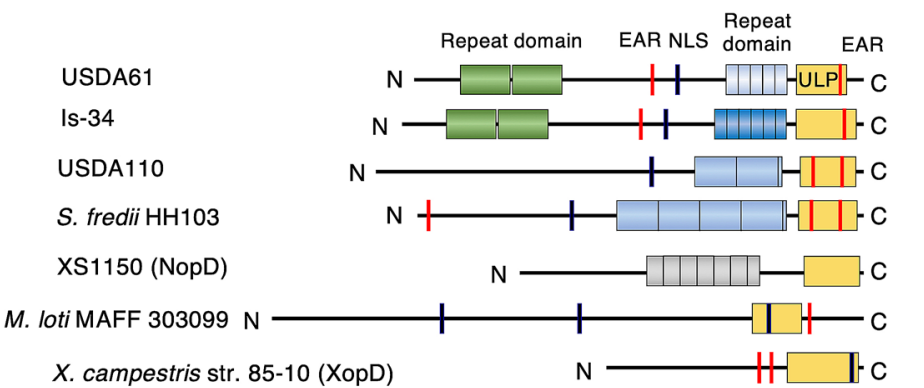

X. campestris str. 85-10 (XopD)

$\overline{100 a a}$

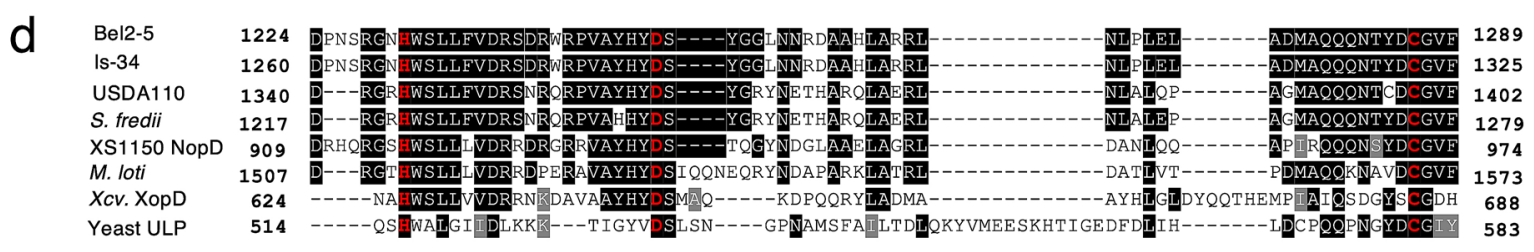

Figure 2. Phylogenetic and In silico analyses of B. elkanii Bel2-5 and its homologs. (a) Neighbor-joining tree of Bel2-5 and its homologs in T3SS-harbouring rhizobia and phytopathogenic bacteria. Boostrap values are given at the nodes with boostrap resampling (1000 replicates of each). (b) Schematic representation of Bel2-5 and XopD from X. campestris str. 85-10. (c) Schematic representation of Bel2-5 functional domains in B. elkanii USDA61, in comparison to its homologs in selected rhizobia and pathogenic bacteria. The repeat domain I and II, EAR motifs, NLS motif and ULP1 domain are shown. (d) Sequence alignment of ULP1 catalytic domain of B. elkanii BEL2-5 and its selected homologs, including: B. japonicum Is-34 (MA20_12780), B. diazoefficiens USDA110 (Bll8244), S. fredii HH103 (SFHH103_04358), Bradyrhizobium sp. XS1150 (NopD), M. loti MAFF 303,099 (Mlr6316), X. campestris pv. vesicatoria str. 85-10 (XopD), and yeast ULP1 (DAA11408.1). H, D and C catalytic core residues are indicated by red. Identical amino acids are shaded in black, and similar amino acids are shaded in gray, respectively.

selected Bel2-5 homologs showed that repeat domains are present only among rhizobia with diverse in length and number of repetitions (Fig. 2c; Supplementary Data 1b).

The C-terminus of Bel2-5 is related with Xcv. XopD contained the small ubiquitin-like modifier (SUMO) protease domain of the C48 peptidase [ubiquitin-like protease 1 (ULP1)] (Fig. 2b,c; Supplementary Data 1b). ULP1, a cysteine protease first isolated from yeast (Saccharomyces cerevisiae) ${ }^{30}$, dynamically and reversibly regulates interactions between SUMO and its protein targets. In-silico analysis specifically on the C-terminal of Bel2-5 (from 1144 to 1328aa) showed that ULP1 domain is widely distributed among prokaryotic and eukaryotic species, yeast (Fig. 2b-d; Supplementary Data 1b,2a). Recently, NopD of Bradyrhizobium sp. XS1150 appears as characterized rhizobial SUMO protease facilitates a symbiotic efficiency ${ }^{31}$. ULP1 active-site function depends on the sequentially ordered catalytic-core triad His-Asp-Cys ${ }^{32}$. Alignment of yeast ULP1 (DAA11408.1) with Bel2-5 putative ULP1 domain and its selected homologs, including XopD and NopD showed that this triad is conserved within Bel2-5 residues (H1231/D1251/C1286) (Fig. 2d; Supplementary Data 2a).

Further analysis of the Bel2-5 coding sequence indicated the presence of two putative ethylene-responsive element-binding factor-associated amphiphilic repression (EAR) motifs (LxLxL) at 715-719 and 1268-1274 aa (Fig. 2b,c; Supplementary Data 1b). In plants, EAR motif-containing proteins are the principal transcriptional regulators responsible for repressing gene transcription, and this process is frequently necessary for modulating plant defence and stress responses ${ }^{33,34}$. Notably, Xcv. XopD possesses two EAR motifs $[(\mathrm{L} / \mathrm{F}) \mathrm{DLN}(\mathrm{L} / \mathrm{F})(\mathrm{x}) \mathrm{P}]$ that are both essential for repressing defence-related genes and maximizing $X c v$. growth in tomato plant ${ }^{35,36}$. The Bel2-5 effector therefore appears to be a modular protein consisting of multiple conserved domains found in T3Es originating from pathogenic and symbiotic bacteria ${ }^{37}$.

Bel2-5 is a T3SS-secreted effector. In rhizobia, the expression of genes encoding T3Es and T3SS components is regulated by the transcriptional activator TtsI and host-derived flavonoids, such as genistein ${ }^{10,11}$. TtsI directly binds to a cis-element $t t s$ box in the promoter region of $t t s$ clusters upon induction by host flavonoids ${ }^{20}$. To determine whether bel2-5 expression is regulated similarly, we examined its expression in the presence/ 
a

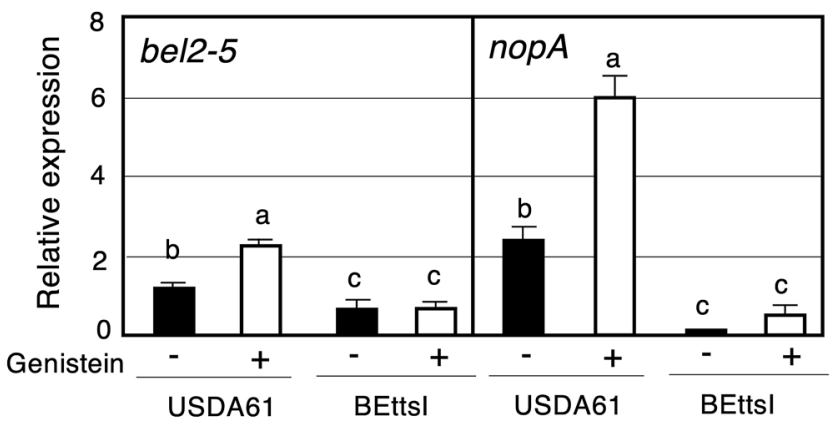

b

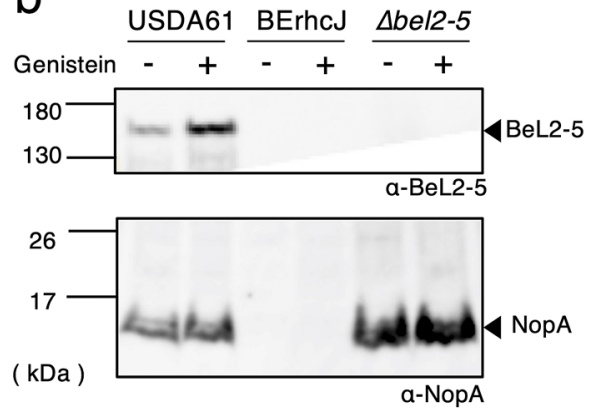

C

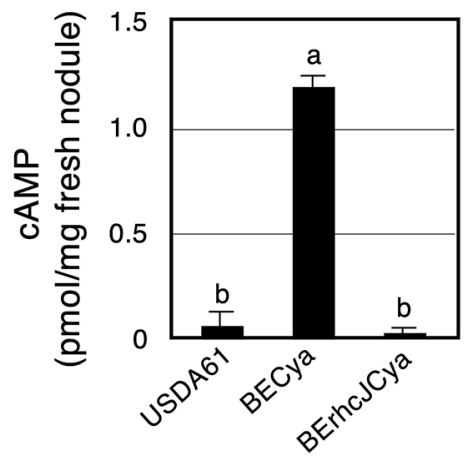

d

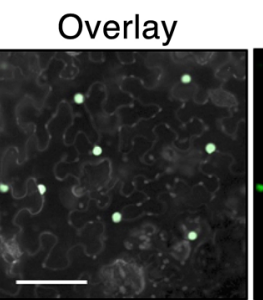

GFP

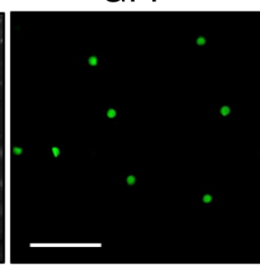

DAPI

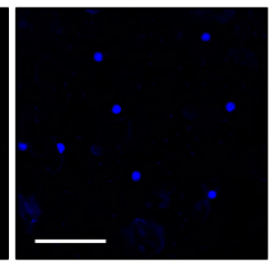

Figure 3. Expression, secretion, translocation and localization of the B. elkanii type III effector Bel2-5. (a) RT-qPCR analysis of bel2-5 and nopA expressions in wild-type and ttsI mutant (BEttsI) of B. elkanii USDA61. Total RNAs were isolated from cultures in the absence $(-)$ or presence $(+)$ of the genistein $(10 \mu \mathrm{M})$ after $48 \mathrm{~h}$ induction. The expression level of each gene was normalized by the ATP synthase $(a t p D)$ gene. Values represent mean \pm SD $(n=3)$. (b) Secretion of Bel2-5 and NopA in the culture supernatant of B. elkanii strains. Secreted proteins from culture supernatants were subjected to immunoblot analysis with the anti-Bel2-5 $(\alpha-B e l 2-5)$ or anti-NopA ( $\alpha-N o p A)$ antibodies. Molecular masses $(\mathrm{kDa})$ of the marker are shown on the left. (c) Translocation of Bel2-5 into soybean nodules. cAMP levels measured from G. max cv. Enrei nodules harvested at $18 \mathrm{dpi}$. Plants were inoculated with B. elkanii USDA61 wild-type, BECya and BErhcJCya. The USDA61 wild-type contained no cya fusion. Values represent mean \pm SD from duplicates measurements. (d) Bel2-5 is targeted to the plant nuclei. Bel2-5 protein fused with eGFP was transiently expressed in $N$. benthamina leaves by Agrobacterium infiltration and visualized $48 \mathrm{~h}$ after by confocal microscopy to determine the subcellular localization. From left to right: an overlay of GFP and DAPI fluorescence spectrums, and the GFP and DAPI fluorescence spectrum, respectively. Staining with DAPI was used to visualize nuclei. On the overlay panel, the DAPI fluorescence was displayed in gray and the contrast and brightness were enhanced in order to be able to visualize the contour of the cells. Scale bars: $100 \mu \mathrm{m}$. Means followed by different letters are significantly different at the 0.05 level by Tukey's method $(\mathbf{a}, \mathbf{c})$.

absence of genistein and TtsI. The expression of nopA, one of the major T3SS machinery components ${ }^{38,39}$, was also quantified as a control. Transcriptional levels of bel2-5 and nopA were significantly increased upon induction with genistein in the WT background. In the ttsI mutant background, the expression of both nopA and bel2-5 genes tested was very weak even in the presence of genistein (Fig. 3a). Collectively, our results confirmed that the expression of bel2-5, similar to that of other T3SS-related genes, was regulated by genistein and TtsI.

To examine the T3SS-mediated secretion of Bel2-5 along with other secreted effectors, an extracellular protein analysis was conducted. For this purpose, the WT USDA61 strain and the bel2-5 deletion and BErhcJ mutant strains were grown in the presence/absence of genistein, and the secreted proteins were harvested at $48 \mathrm{~h}$ postinduction. The generated secreted proteins were used for western blotting analysis of Bel2-5 using an anti-Bel2-5 antibody and led to the detection of a predicted Bel2-5 band $(\sim 146 \mathrm{kDa})$, which was observed only in USDA61 cultures with the enhancement of band intensities by the addition of genistein, and none were detected in the T3SS mutant (BErhcJ) and bel2-5 mutants (Fig. 3b). To exclude the possibility that the deletion of bel2-5 impacts the secretion of other effectors, in other words, that Bel2-5 participates in the functioning of the T3SS machinery, we also analysed the presence of the NopA in the culture supernatants of USDA61, the Bel2-5 mutant and T3SS machinery deficiency (BErhcJ) mutants with an anti-NopA antibody. As shown in Fig. 3b, NopA was detected in both the WT strain and the bel2-5 mutant but not in the BErhcJ mutant, demonstrating that the secretion of the other T3Es was not blocked in the bel2-5 mutant. Collectively, these data confirm that Bel2-5 is a T3SS-secreted protein. Although the signal intensity of the band corresponding to the Bel2-5 or NopA protein was altered by the addition of genistein, their presence could also be detected in cultures without genistein. These results are consistent with previous results ${ }^{14,22}$, suggesting that T3SS is activated in a genistein-independent manner 
a

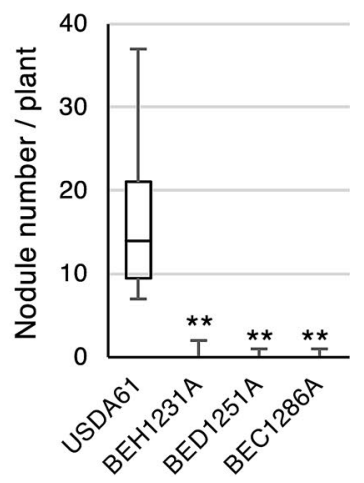

b

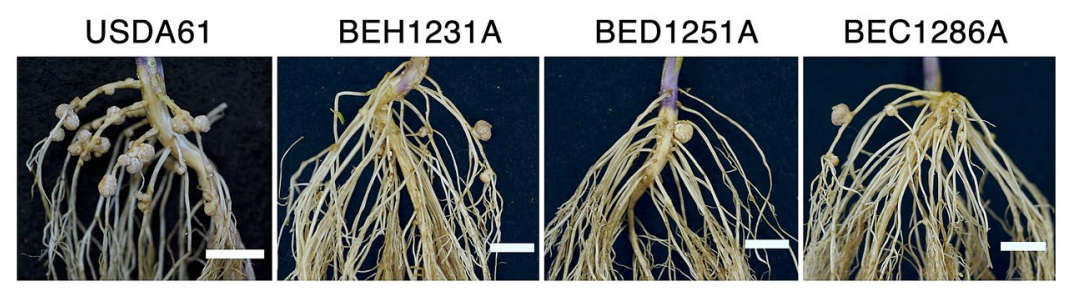

Figure 4. Nodulation of G. $\max \mathrm{cv}$. En1282 ( $n f r 1$ mutant soybean) inoculated with Bel2-5 ULP1 domain mutant strains. (a) The number of nodules formed on En1282 inoculated with wild-type and the mutants of predicted catalytic core of Bel2-5 ULP1 at 30 dai. Values represent mean \pm SD $(n=15$, from three independent inoculation tests). ${ }^{* *} p<0.001$ by two-tailed Student $t$-test compared to USDA61 wild-type. (b) Roots of En1282 inoculated with wild-type and the mutants of predicted catalytic core of Bel2-5 ULP1 were photographed at 30 dai. Scale bars: $1 \mathrm{~cm}$.

under the test conditions. Therefore, unidentified regulators or inducers may be involved in the regulation of the USDA61 tts genes.

To confirm that Bel2-5 is translocated into host cells, we used an adenylate cyclase (Cya) reporter system. Here, the Bel2-5 C-terminus was fused to the calmodulin-dependent adenylate cyclase domain of Cya, and thus, if bel2-5cya was translocated into cells, cAMP synthesized by Cya due to calmodulin, which is present only in eukaryotic cells, could be detected ${ }^{40}$. The bel2-5cya hybrid was introduced into the WT USDA61 and BErhcJ strains to generate BECya and BErhcJCya, respectively, and cAMP levels were measured from fresh Glycine max cv. Enrei nodules inoculated with the mutants at 18 days post-inoculation (dpi) (Fig. 3c); cAMP accumulation was substantially increased in nodules infected with BECya but not BErhcJCya or USDA61 lacking cya (Fig. 3c), which confirmed that Bel2-5 was translocated into plant cells and that the T3SS machinery was essential for its translocation.

The presence of an NLS motif at 788-796 aa in Bel2-5 indicated potential nuclear translocation of the protein (Fig. 2b,c; Supplementary Data 1b). To check whether Bel2-5 targets the nuclei of plant host cells, we constructed a Bel2-5 C-terminally fused with enhanced GFP (Bel2-5-eGFP) and transiently expressed it in Nicotiana benthamiana leaves using Agrobacterium tumefaciens-mediated transformation. Confocal analysis performed $48 \mathrm{~h}$ after infiltration confirmed the nuclear localization of Bel2-5, as the GFP fluorescence signal colocalized specifically with the DAPI signal (a nuclear staining) in the nucleus of the cells (Fig. 3d). This result indicate that Bel2-5 is translocated into the host cell and targeted to the plant nucleus.

The mutation in ULP1-like domain of Bel2-5 impairs nodule formation on $n$ fr1 mutant soybean. Because Bel2-5 was found to carry a C-terminal cysteine protease (ULP1-like) domain (Fig. 2b-d; Supplementary Data $1 \mathrm{~b}$ and $2 \mathrm{a}$ ), we suspected that the domain might play a role during symbiosis. The effector protease functions reveal that proteolysis of a host substrate is an important strategy employed by pathogens to alter host physiology $y^{41}$, and thus, proteolysis may have an essential role in symbiont-plant interactions. To examine whether the ULP1-like domain is required for Bel2-5-promoted symbiosis, we performed substitution analysis of predicted triad catalytic residues, His (H1231), Asp (D1251), and Cys (C1286), with Ala and named these: BEH1231A, BED1251A, and BEC1286A, respectively. A nodulation test on En1282 plants revealed that in contrast to the WT USDA61, which formed nodules, the mutants of ULP1 catalytic residues had drastically reduced nodulation capacity (Fig. 4). These results suggest that ULP1 domain of Bel2-5 effector is required for nodulation on $n f r 1$ mutant soybean.

Heterologous Bel2-5 expression confers hijacking activity to other rhizobia. Bradyrhizobium sp. ORS3257 possesses a functional T3SS and forms nodules on A. indica in a T3SS-dependent manner due to secretion of the ErnA effector ${ }^{19}$. We investigated in this study whether this strain was also able to nodulate En1282 soybean roots. As shown in Fig. 5a,b, En1282 plants inoculated with the ORS3257 strain did not form nodules, suggesting that USDA61 and ORS3257 differ in their mode of action to induce NF-independent symbiosis in these two host plants. ErnA and Bel2-5 share some similarities with a conserved 85 aa domain of unknown function displaying 35\% identity (located at positions 706 and 790 aa in Bel2-5) (Supplementary Data 2b), but the similarities stop at this level and ErnA lacks of ULP1-like domain that we confirmed important for Bel2-5 triggering nodules formation in $n f r 1$ soybean roots. To verify Bel2-5 as the genuine effector that activates nodulation signalling in $n f r 1$ soybean plants, bel2-5 was introduced into ORS3257 to generate ORS3257::bel2-5, which was then used (together with WT ORS3257) for En1282 nodulation tests. The introduction of bel2-5 conferred the ability of ORS3257 to form nodules on En1282 soybean roots (Fig. 5). Nodule sections displayed 
a

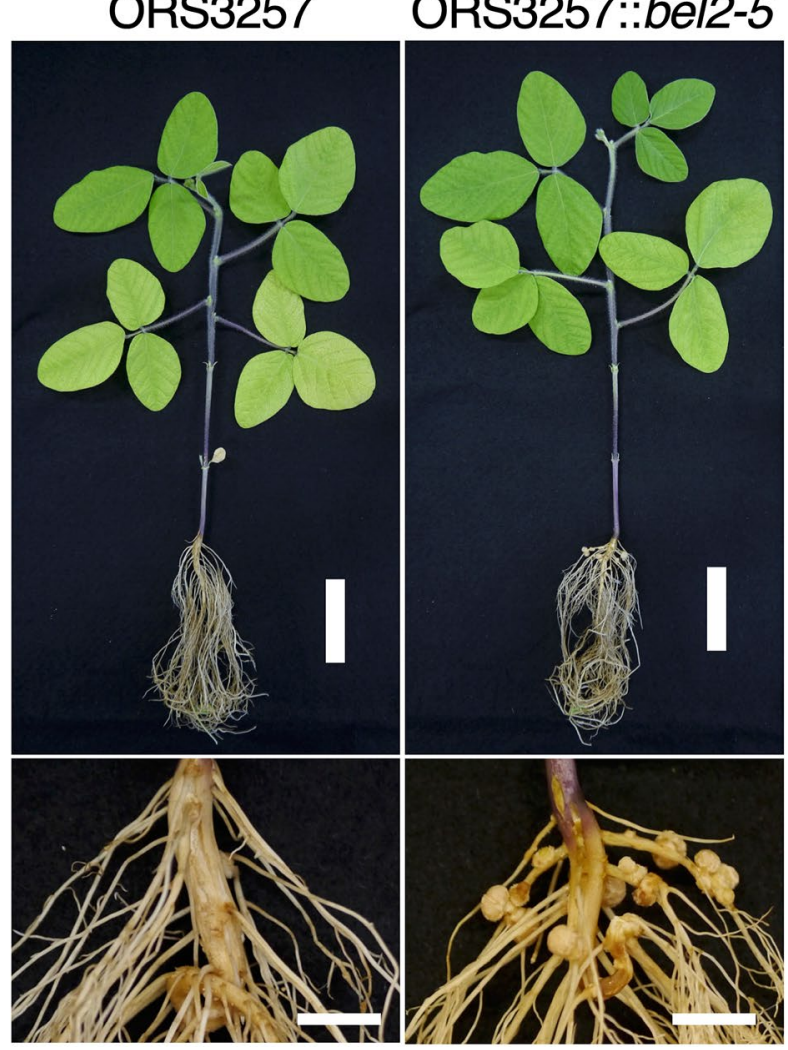

b

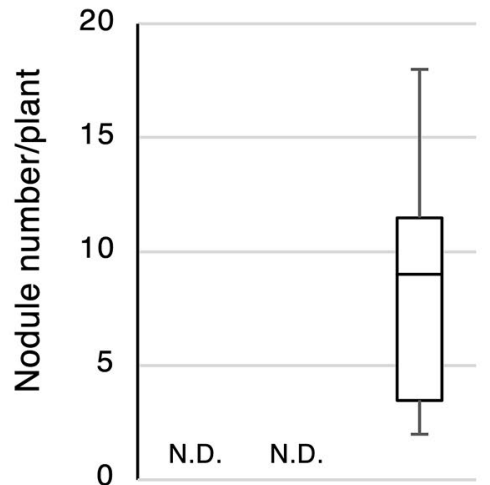

c

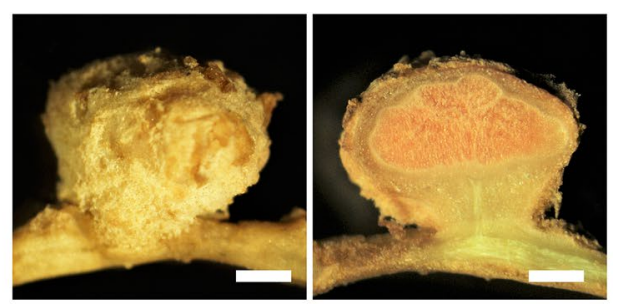

Figure 5. Bel2-5 confers hijacking activity to another rhizobia. (a) G. max cv. En1282 ( $n f r 1$ mutant soybean) inoculated with Bradyrhizobium sp. ORS3257 wild-type and with insertion of bel2-5 gene, designed as ORS3257::bel2-5. Whole plants (scale bars: $5 \mathrm{~cm}$ ) and root nodules (scale bars: $1 \mathrm{~cm}$ ) were photographed at 30 dpi. (b) The number of nodules formed on En1282 inoculated with uninoculated (water), ORS3257 and ORS3257::bel2-5. Nodules were counted at 30 dai. Values represent mean \pm SD $(n=18$, from three independent inoculation tests). N.D.: Not detected. (c) nodules section of En1282 inoculated with ORS3257::bel2-5. Scale bars: $1 \mathrm{~mm}$.

the characteristic pink colour of leghemoglobin, suggesting that the nodules elicited by ORS3257::bel2-5 were functional and able to fix nitrogen, as are those induced by USDA61 (Fig. 5c). These results indicate that a gain of function in the ability to nodulate soybean plants in the absence of NF signalling is possible by transferring bel2-5 to another Bradyrhizobium strain, confirming that Bel2-5 is the key effector governing this symbiotic interaction.

Bel2-5 regulates soybean symbiosis- and defence-related genes. We previously reported that the soybean nodulin genes were upregulated by the USDA61 T3SS in the absence of NF signalling ${ }^{17}$. To enhance our understanding of the soybean genes whose expression is modulated by Bel2-5, we performed RNA sequencing (RNA-seq) and compared the transcriptome of En1282 roots inoculated with WT and bel2-5 deletion mutant strains (Fig. 6). At least 153 and 76 genes were found to be upregulated (fold change $\geq 1.5$ ) and downregulated (fold change $\leq$ 0.5), respectively, in soybean roots during interaction with WT B. elkanii compared with those of genes in soybean roots during interaction with the bel2-5 mutant. These differentially expressed genes (DEGs) were sorted using the gene-function classification system Gene Ontology (GO) for biological process, molecular function, and cellular components (Fig. 6a). Some representative DEGs were also validated by qRT-PCR analysis (Supplementary Fig. 2).

The biological processes (BPs) associated with upregulated DEGs were carbohydrate metabolic process, cell wall modification, and xyloglucan metabolic process (Fig. 6b). Genes belonging to these groups encode pectinesterase-family enzymes, $\beta$-1,3-endoglucanase, exoglucanase/xylanase-like protein, polygalacturonase, and L-lactate dehydrogenase-like protein (Fig. 6c; Supplementary Table 2). The upregulation of these genes is probably related to $B$. elkanii infection, which requires root cell wall loosening necessary for bacterial internalization. Of interest in the identified upregulated genes is the small number of soybean root DEGs annotated in the cytokinin biosynthetic process, including 2 homologues of adenylate isopentenyltransferase 5 (IPT5) and cytokinin riboside 5'-monophosphate phosphoribohydrolase (LOG1-like) (Fig. 6b,c; Supplementary Table 2). One gene homologous to cytokinin hydroxylase (CYP735A), annotated in the BP oxidation-reduction process (Fig. 6c; Supplementary Table 2), was also upregulated during USDA61 infection. The CYP735A gene converts isopentenyl adenine (iP) into trans-zeatin (tZ) nucleotides in the cytokinin synthesis pathway ${ }^{42}$. The positive role 
a Gene Ontology (GO) classification
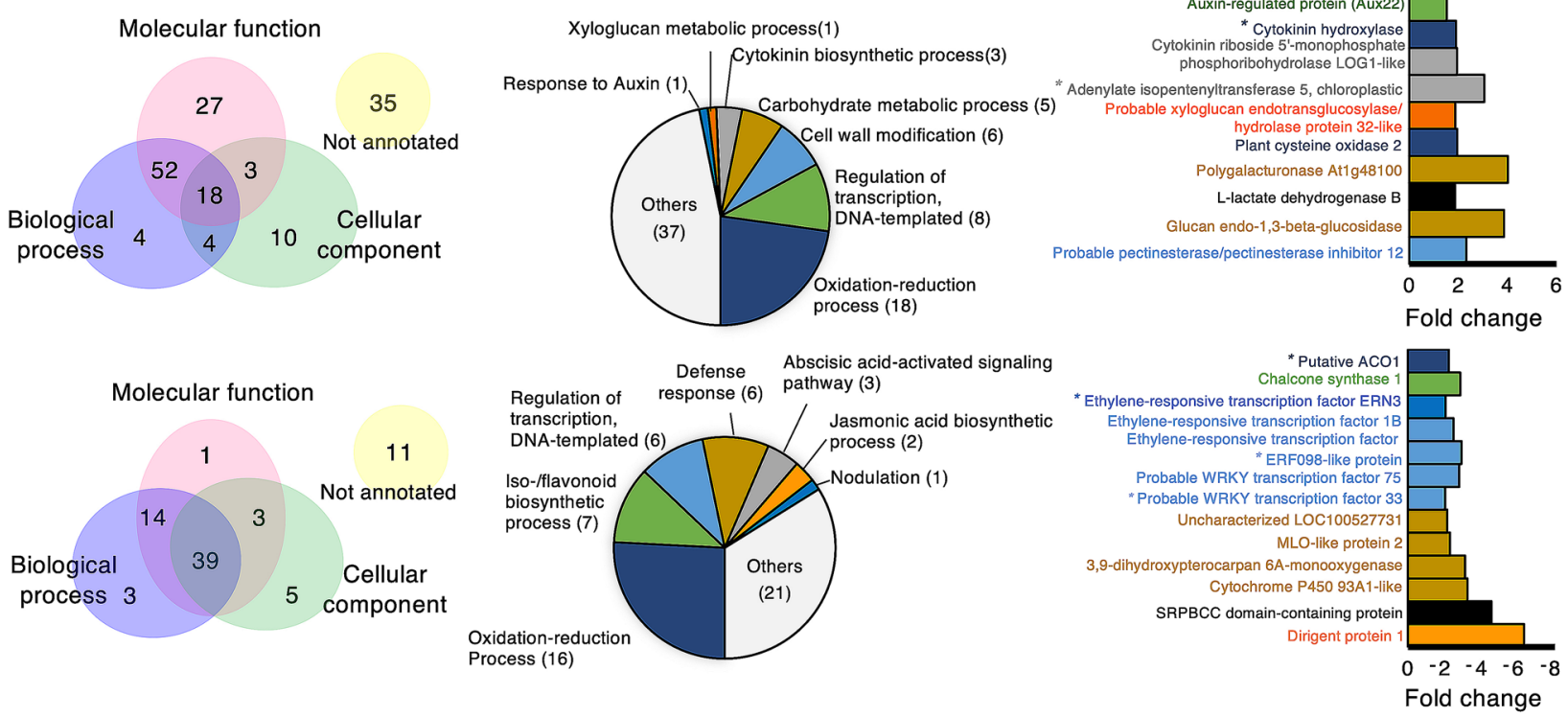

(Ratu et al., 2020)

Figure 6. Soybean genes differentially expressed by the B. elkanii T3 effector Bel2-5. (a) Gene ontology classification of the soybean genes up- or down-regulated during interaction with B. elkanii wild-type as compared with bel2-5 deletion mutant. To detect the soybean genes that were up-regulated and down-regulated, RNAseq fold in the roots of En1282 inoculated with USDA61 wild-type were compared to the roots of En1282 inoculated with bel2-5 deletion mutant. The soybean genes with signal intensities $\geq 1.5$-fold and $\leq 0.5$-fold were considered to be up-regulated and down-regulated, respectively. (b) GO classification on biological process (GO_BP) of soybean DEGs. Several DEGs were annotated into multiple classes of GO_BP. (c) Characteristics of several down-regulated and up-regulated of soybean genes. The bar's color represents in where gene was annotated in GO_BP. The bar with black color represents gene was annotated into multiple classes of GO_BP. See Supplementary Table 3 and 4 for the details $(\mathbf{b}, \mathbf{c})$. Asterisk $\left(^{*}\right)$ means representative DEGs were validated with qRT-PCR (Supplementary Fig. 2).

of sufficient cytokinin levels in nodule organogenesis of soybean has been well documented ${ }^{43,44}$. It is therefore tempting to assume that Bel2-5 enhances nodulation in $n f r 1$-mutant soybean roots by upregulating genes related to cytokinin biosynthesis.

Conversely, several downregulated DEGs were linked to iso-/flavonoid biosynthetic processes (6 genes homologous to chalcone synthase $(\mathrm{CHS})$ ), and one gene is homologous to 2-hydroxyisoflavanone synthase (Fig. 6b,c; Supplementary Table 3). In plants, the expression of these genes is involved in the salicylic acid defence response against bacterial or fungal infection ${ }^{45}$. Six soybean DEGs were annotated as defence response genes, including two genes homologous to SRPBCC domain-containing protein, two genes homologous to cytochrome P450 93A1-like (CYP93A1), one gene homologous to MLO-like protein, and one uncharacterized gene (LOC100527731) (Fig. 6b,c; Supplementary Table 3). The SRPBCC domain is a lipid/sterol-binding domain that binds various ligands via a hydrophobic pocket and may interact with salicylic acid (SA) pathway to regulate defense response ${ }^{46,47}$. Likewise, $M L O$-like gene belonging to plant-specific calmodulin-binding protein regulates cell death and defense response against powdery mildew. At least 20 genes encoding MLOs have been identified in soybean plant ${ }^{48-50}$.

We also found the downregulation of some transcription factors known to be involved in plant immunity responses against pathogen attack and stress conditions, including two homologues of putative WRKY transcription factors (WRKY33/75) and ethylene-responsive factors, ERF1b and ERF98 (Fig. 6c; Supplementary Table 3). Three homologues of the aminocyclopropane-1-carboxylate oxidase (ACO) gene (annotated in the oxidation-reduction process) were also downregulated (Fig. 6c; Supplementary Table 3). The ACO gene is involved in the biosynthesis of ethylene, a plant hormone that regulates many developmental and physiological processes, including defence responses ${ }^{51}$. Collectively, these results suggest that USDA61 also enhances symbiosis with $n f r 1$ mutant soybean through dampening of host immunity to promote rhizobial infection in the early stage of the interaction. Moreover, one gene homologous to ERF required for nodulation (ERN3) was downregulated during USDA61 infection (Fig. 6c; Supplementary Table 3). ERN3 negatively regulates nodulation by acting as a putative repressor of the activator of NF-box-containing targets ERN1/ERN2 essential for Medicago truncatula $(M t) E N O D 11$ expression $^{52}$. This result suggests that ERN3 downregulation might also promote the activation of nodulation-related genes on $n f r 1$ mutant soybean. 


\section{Discussion}

The Bradyrhizobium sp. ORS3257, due to its T3SS, can nodulate Aeschynomene plants in an NF-independent manner ${ }^{18,19}$. Recently, it was reported that this symbiosis relies on a set of effectors, including NopM1, NopP1, NopAB, and NopT, that play complementary and synergetic roles together with ErnA, which acts as the major effector triggering nodulation ${ }^{19}$. Here, we showed that during the T3SS-dependent symbiosis between Bradyrhizobium USDA61 and $n f r 1$ soybean plants, another set of effectors is required, including ErnA, NopL, Bel2-5 and possibly other effectors not yet identified. Besides, we can not exclude the possibility that B. elkanii NFs also play roles in the nodulation of $n f r 1$ soybean. We previously showed that $B$. elkanii nodC mutant induced fewer nodules than WT B. elkanii ${ }^{17}$. In addition, it has been shown that $n f r 1$ mutant soybeans retained the ability to suppress microbe-associated molecular pattern (MAMP)-triggered immunity upon addition of $\mathrm{NF}^{53}$. These results suggest that cocktail of effectors and NFs in B. elkanii harmoniously promote nodulation on $n f r 1$ mutant soybean.

NopL has been reported to promote nodulation in some rhizobium-legume symbioses. NopL has several phosphorylated sites, and its phosphorylation in plant cells interferes with the MAPK signalling pathway, suppressing the defence response $\mathrm{e}^{13,54,55}$. The ErnA protein was shown to be a remarkable effector that has a direct function to induce cell division to initiate nodule organogenesis. Transgenic Aeschynomene lines overexpressing ernA form root or nodule-like structures all along the $\operatorname{root}^{19}$. However, during the symbiotic interaction between the USDA61 strain and soybean plants, the dominant effector is not ErnA, which is at the centre of this symbiosis, but the effector Bel2-5, which was identified as the key factor inducing nodulation on $n f r 1$ mutant soybean (Fig. 1).

Homology searches identified sequence similarities between Bel2-5, the XopD of Xanthomonas spp. and NopD of Bradyrhizobium sp. XS1150 (Fig. 2). The XopD effector was originally identified from Xcv., which causes spot disease on tomato and pepper plants, while the NopD restricts nodulation of Tephrosia vogelii by Bradyrhizobium sp. XS1 $150^{31,56}$. In the present study, we showed that the Bel2-5 positively regulates the activation of soybean nodulation signalling. Bel2-5 and XopD share a conserved ULP1-like domain in their C-terminal region but show no homology within the N-terminal and central regions. The XopD N-terminus contains a DNA-binding domain, while the Bel2-5 N-terminus comprises a repeat sequence that is conserved only among rhizobia and an additional central region of 85 aa that is conserved in the ErnA effector (Fig. 2b; Supplementary Data $1 \mathrm{~b}$ and $2 \mathrm{~b}$ ). These differences between Bel2-5 and XopD could underlie their distinct host specificities and target substrates, which lead to different phenotypes (symbiosis or pathogenesis) during interaction with their host plants.

A striking similarity between Bel2-5, NopD, and XopD is the fact that defective on ULP1 domain impairs their interaction with host plants. The respective mutations of this domain abrogated the capacity of USDA61 to form nodules (Fig. 4), while it abolished the ability of X. campestris and Bradyrhizobium sp. XS1150 to elicit leaf necrosis in $N$. benthamiana ${ }^{31,35}$. The members of the ULP1 protease family were reported to modulate diverse functions of various molecular processes, including gene expression, chromatin remodelling, subcellular localization, and genome maintenance ${ }^{57}$. Soybean harbours at least 13 SUMO proteases, among which $G m B 2 d$ and $G m E S D 4 a / b / c$ are highly expressed inside root nodules ${ }^{58}$, however, the involvement of SUMO proteases in nodulation remains unclear. Meanwhile, Xcv. XopD has been reported to perturb SUMO pathway regulation for pathogenic purposes ${ }^{29,59}$. In particular, the deSUMOylation of the tomato transcription factor SIERF4 by XopD permits the suppression of ethylene production and promotes $X c v$. infection in tomato plants ${ }^{59}$. Interestingly, our RNA-seq analysis showed that the expression of the soybean ACO gene, which catalyses the final step in ethylene biosynthesis, was repressed by USDA61, but not by the bel2-5 mutant (Fig. 6c; Supplementary Table 3). Two ethylene transcriptional regulators, ERF1b and ERF98, together with probable TF WRKY33/75, that govern plant defence responses were also suppressed (Fig. 6c; Supplementary Table 3). Hence, it is tempting to speculate that similar to XopD, Bel2-5 may promote USDA61 infection by interfering host signalling, via ethylene-related genes or host-dependent immune system. Further study of identification Bel2-5 host targets and biochemical analysis would not only reveal the molecular basis of how Bel2-5 promotes nodulation by modulating the host SUMO system but also clarify the missing link between the SUMO system and nodulation.

Finally, we observed an overexpression of cytokinin biosynthetic genes in plants inoculated with the WT strain, in contrast to the situation in those inoculated with the bel2-5 mutant (Fig. 6b,c; Supplementary Table 2). It is tempting to speculate that transcriptional changes in cytokinin biosynthesis genes influence the downstream targets of NF perception, ENOD40 and NIN, to stimulate nodule development. ENOD40 has been shown to be induced in the modest mode, whereas NIN expression was increased at the steady-state level in Lotus japonicus roots by external application of cytokinin ${ }^{60}$. Likewise, spontaneous nodule-like structure formation was triggered by overexpression of the cytokinin complete gene set, $L j I P T 3, C y p 735 a$, and $\operatorname{LjLog} 4^{61}$. Although the molecular mechanism underlying the induction of cytokinin biosynthetic related genes remains unclear, this effect might be related to the N-terminus of Bel2-5, which has little similarity with XopD (Fig. 2b), considering that XopD does not have this activity. Further analysis of N-terminal repeat domains that are well conserved among only rhizobia could provide insight into the unique symbiotic function of Bel2-5 and how they differentiate Bel2-5 from other pathogenic homologues.

In conclusion, our study identified that the USDA61 Bel2-5 effector enhances soybean nodule formation by activating soybean nodulation signalling. We propose a model of modulated by USDA61 T3Es in Fig. 7. The Bel2-5 effector likely interferes with the NF-dependent signalling pathway at least at two levels: (1) by modulating cytokinin biosynthesis-related genes that favouring nodule organogenesis, and (2) by repressing ethylene biosynthesis-related genes and host-dependent defence responses that are deleterious to the rhizobial infection. It should be noted that these DEGs may contain secondary and tertiary indirect effects as well as Bel2-5 direct effects. Two other effectors, ErnA and NopL, might be required for efficient and robust nodule development. Identification of the host targeted protein(s) and characterization of the Bel2-5 unique domains would provide 


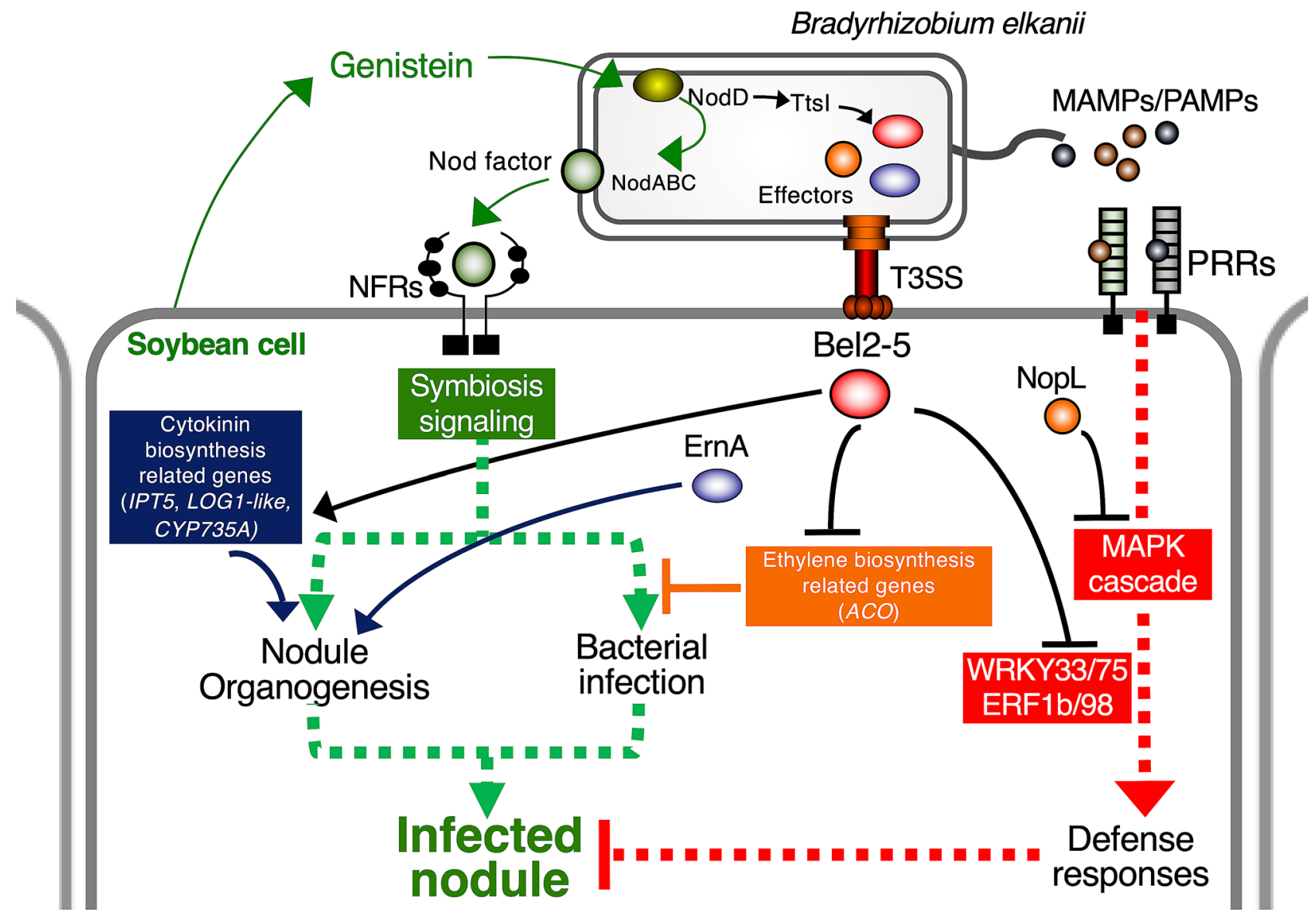

Figure 7. Proposed model for nodulation signaling and modulation by Bradryhizobium elkanii type III effector Bel2-5. A soybean root-derived genistein induces the production of Nod factors (NFs) in B. elkanii. Recognition of NFs by NF receptors triggers a signaling cascade leading to nodulation. The genistein also induces B. elkanii to inject effector proteins into soybean cells. NopL suppress plant defense responses by modulating the MAPK cascade. The ErnA effector triggers nodule organogenesis by a yet unknown mechanism. Bel2-5 effector likely interferes with the nodulation signaling pathway at least at two levels: (1) by modulating cytokinin biosynthesisrelated genes that promoting nodule organogenesis and (2) by repressing ethylene biosynthesis-related genes and host-dependent defence responses that are deleterious to the bacterial infection. It should be noted that these DEGs may contain secondary and tertiary indirect effects as well as Bel2-5 direct effects.

insights into how rhizobia have employed and customized T3Es for symbiotic purposes during co-evolution between plants and bacteria.

\section{Materials and methods}

Bacterial strains and growth conditions. The bacterial strains and plasmids we used are listed in Supplementary Table 4. B. elkanii strains and A. tumefaciens GV3101 were grown at $28^{\circ} \mathrm{C}$ in Arabinose-Gluconate $(\mathrm{AG})$ medium $^{62}$ supplemented with appropriate antibiotics $(50 \mu \mathrm{g} / \mathrm{mL}$ polymyxin, $200 \mu \mathrm{g} / \mathrm{mL}$ kanamycin, $200 \mu \mathrm{g} / \mathrm{mL}$ streptomycin, and 100 or $200 \mu \mathrm{g} / \mathrm{mL}$ spectinomycin). Escherichia coli strains were grown at $37^{\circ} \mathrm{C}$ in Luria-Bertani medium ${ }^{63}$ supplemented with appropriate antibiotics $(50 \mu \mathrm{g} / \mathrm{mL}$ kanamycin, $50 \mu \mathrm{g} / \mathrm{mL}$ streptomycin, and $10 \mu \mathrm{g} / \mathrm{mL}$ tetracycline). All conjugation processes were performed on Peptone Salt Yeast Extract (PSY) medium ${ }^{64}$.

Antibodies. To obtain anti-NopA and anti-Bel2-5 antisera, a rabbit was immunized with a synthesized peptide corresponding to a partial sequence of NopA (N-RSMLLRTVTTELQTTKKAADERVQ-C) or Bel2-5 (N-EALRSGNAAERTAK-C and N-DAAELRPAKRPRTL-C), which were coupled to carrier proteins prior to immunization, according to the manufacturer's protocols (Eurofins Japan, Tokyo, Japan).

Plasmid construction, mutagenesis, and complementation. All constructions made in this study are listed in Supplementary Table 4, which includes the primers and cloning strategies. For the construction of insertional mutants (obtained by single crossing-over), a 350- to 600-bp internal fragment of the target gene was amplified by PCR and cloned into the nonreplicative plasmid pSUPSCAKm. For the construction of in-frame deletion mutant of bel2-5 ( $\Delta$ bel2-5), 800- to 900-bp PCR fragments corresponding to the upstream and down- 
stream flanking regions of the bel2-5 were merged by overlap extension PCR and cloned into pK18mobsacB. The deletion mutant was selected on AG medium supplemented with $10 \%$ sucrose. Sucrose-resistant clones were checked for loss of kanamycin resistance from the pK18mobsacB plasmid, and kanamycin-sensitive clones were screened by PCR for the deletion of the bel2-5.

For bel2-5 complementation, 4.5-kb DNA fragments containing bel2-5 with its promoter sequence were PCR amplified and cloned into pBjGroEL4::DsRed2. The constructed plasmid (pBjGroEL4::bel2-5) was mobilized into the $\Delta$ bel2-5 by conjugation. Integration of the bel2-5 gene into the chromosome of $\Delta b e l 2-5$ was confirmed by antibiotic resistance, PCR and sequencing.

The substitution of Bel2-5 ULP1 putative catalytic residues (H/D/C) were performed using a QuickChange Lightning Site-Directed Mutagenesis Kit (Agilent Technologies). First, a partial fragment of bel2-5 (1474 bp) from the $3^{\prime}$-end (C-terminus) was amplified by PCR and cloned into the plasmid pS18mob to generate pS18mobbel2-5ULP1, which was then used as the template for generating plasmid carrying a substitution of $\mathrm{H} / \mathrm{D} / \mathrm{C}$ into alanine using specific primer sets. The constructed plasmids were confirmed using sequencing analysis and then integrated into WT USDA61 through single homologous recombination. The constructed mutants were screened for antibiotic resistance and confirmed using sequencing analysis.

Nodulation tests. Soybean seeds (En $1282^{65}$ and Enrei) were surface-sterilized and germinated as described $^{20}$; 2-days-old germinated seedlings were transferred into a plant box (CUL-JAR300; Iwaki, Japan) containing sterilized vermiculate and inoculated with $1 \times 10^{7}$ cells $/ \mathrm{mL}$ B. elkanii strains or sterilized water (mock treatment). Plants were cultivated in a plant growth chamber at $25^{\circ} \mathrm{C}$ and $70 \%$ humidity under a $16 / 8$-h day/ night regimen; appropriate amounts of B\&D nitrogen-free solutions ${ }^{66}$ were added for watering the plants. Nodule number, nodule weight, and plant fresh weight were measured at 30 dai.

Bacterial RNA extraction and expression analysis. RNA was extracted from bacterial cells as described ${ }^{14}$. Briefly, rhizobial strains were grown at $28^{\circ} \mathrm{C}$ in $\mathrm{AG}$ medium supplemented with appropriate antibiotics $(50 \mu \mathrm{g} / \mathrm{mL}$ polymyxin and $200 \mu \mathrm{g} / \mathrm{mL}$ streptomycin $)$, and after 3 days, bacterial cells $\left(\mathrm{OD}_{600}=0.4\right)$ were transferred to new medium supplemented with or without $10 \mu \mathrm{M}$ genistein. After $48 \mathrm{~h}$ of growth, bacterial cells were harvested for total RNA extraction using TRI Reagent (Cosmo Bio Co., Ltd.). Total bacterial RNA was treated with Recombinant DNase I (RNase-free) (Takara Bio Co., Ltd.) and purified using phenol-chloroform extraction.

The extracted RNA samples were used as the template for cDNA synthesis in a final volume of $20 \mu \mathrm{L}$ (containing $400 \mathrm{ng}$ of total RNA) by using the SuperScript III First-Strand Synthesis System for RT-PCR (Invitrogen). The RT-qPCR mixture comprised of $1 \mu \mathrm{l}$ of tenfold diluted cDNA template, $1 \mu \mathrm{l}$ of primer solution (containing $2 \mu \mathrm{M}$ of each forward and reverse primers), $6.6 \mu \mathrm{l}$ of Milli-Q water and $10 \mu \mathrm{l}$ of $2 \times$ KAPA SYBR Fast qPCR Master Mix (KAPA Biosystems). Thermal cycling condition consisted of $2 \mathrm{~m}$ in at $50{ }^{\circ} \mathrm{C}, 30 \mathrm{~s}$ at $95^{\circ} \mathrm{C}$, and 40 cycles of $5 \mathrm{~s}$ at $95^{\circ} \mathrm{C}$ and $30 \mathrm{~s}$ at $60^{\circ} \mathrm{C}^{16}$. RT-qPCR was analyzed using a StepOne Real-Time PCR System (Applied Biosystems) with the primer set listed in Supplementary Table 4. Each sample consisted of three biological replicates and two technical replicates. Transcript levels of bel2-5 were normalized to those of the housekeeping gene atp $D^{67}$, which were measured in the same samples.

Purification and analysis of extracellular proteins. For isolation of extracellular proteins, AG medium was inoculated with 1:100 dilution from a B. elkanii preculture. Extracellular proteins of B. elkanii strains were recovered from $500 \mathrm{~mL}$ of cultures $(+/-10 \mu \mathrm{M}$ genistein) grown on an incubator shaker $(200 \mathrm{rpm})$ for $48 \mathrm{~h}$. Cultures were centrifuged in two steps $\left(1 \mathrm{~h}, 4000 \times \mathrm{g}, 4^{\circ} \mathrm{C}\right.$, and then $\left.30 \mathrm{~min}, 8000 \times \mathrm{g}, 4^{\circ} \mathrm{C}\right)$, the obtained supernatants were lyophilized to complete dryness, and extracellular proteins were isolated as described ${ }^{68}$. For Bel2-5 and NopA immunodetection, proteins were separated on 4-15\% SDS-PAGE gradient gels, electroblotted onto Polyvinylidene Difluoride Membranes (Bio-Rad, USA), blocked with Western Blot Blocking Buffer (Fish Gelatine; Takara, Japan), and incubated with a raised antiserum as the primary antibody (Bel2-5 $=1: 1000$ or NopA $=1: 4000$ dilution) and then with a horseradish peroxidase-conjugated goat anti-rabbit immunoglobulin G antiserum (secondary antibody, 1:40,000). An ECL Prime Kit (GE Healthcare, UK) was used to develop the membranes, and the Bel2-5 band was detected using a LAS-3000 Luminescent Image Analyzer (Fujifilm, Japan).

Adenylate cyclase (Cya) assay. A bel2-5cya fusion was constructed by cloning the C-terminal-coding region of bel2-5 without the stop codon ( $575 \mathrm{bp})$; the fragment was PCR amplified and cloned into the plasmid pSLC5Sm ${ }^{14}$ to generate the plasmid pSLC5Sm-bel2-5cya, which was confirmed through sequencing analysis. Integration of the bel2-5cya fusion into WT USDA61 and its derivative BErhcJ mutant was screened based on antibiotic resistance and confirmed using PCR.

The cAMP levels were quantified from G. max cv. Enrei root nodules collected randomly from three separate plants at $18 \mathrm{dpi}$. The collected root nodules were frozen in liquid nitrogen and ground into a fine powder, which was resuspended in a $10 \times$ volume of $0.1 \mathrm{M}$ hydrochloric acid solution (per nodule weight). The suspension was centrifuged, and the supernatant was used for cAMP measurement by using a Cyclic AMP (Direct) Enzyme Immunoassay (EIA) Kit (Cayman Chemical Co., Ann Arbor, MI, USA) according to the manufacturer's instructions. Each sample was diluted for measurement of the cAMP concentration in the detection range of the standard assay.

Subnuclear localization analysis. The ORF of bel2-5 without the stop codon was PCR amplified and cloned into the Gateway Entry Vector pCR8/GW/TOPO (Thermo Fisher). The PCR product was recombined downstream of a $35 \mathrm{~S}$ promoter into the destination vector pB7FWG2.0 (https://gateway.psb.ugent.be) with a 
C-terminal GFP tag. The recombinant vector was transformed into competent cells of $A$. tumefaciens strain GV3101. Leaves from four-week-old $N$. benthamiana plants were infiltrated using a needleless syringe containing bacteria resuspended in infiltration buffer $(10 \mathrm{mM} \mathrm{MgCl} 2 ; 10 \mathrm{mM} \mathrm{MES}-\mathrm{KOH} \mathrm{pH} \mathrm{5.6;150} \mu \mathrm{M}$ acetosyringone) and adjusted to OD600 $=0.5$.

At $48 \mathrm{~h}$ following A. tumefaciens infiltration, $N$. benthamiana leaf samples were incubated in $5 \mu \mathrm{g} / \mathrm{mL}$ DAPI solution (4',6-diamidino-2-phenylindole; Sigma) for $30 \mathrm{~min}$. Localization of fluorescently labelled bel2-5 was observed with a confocal microscope (CarlZeiss LSM700). GFP was excited at $488 \mathrm{~nm}$, with emission signal collection at 490-530 nm, while DAPI was excited at $405 \mathrm{~nm}$, with emission signal collection at 410-470 $\mathrm{nm}$. Images were obtained using ZEN2008 software (Zeiss).

Soybean RNA-Seq and qRT-PCR analysis. Sample preparation and cDNA library construction. For RNA extraction from soybean roots, seeds were surface-sterilized, germinated at $25{ }^{\circ} \mathrm{C}$ for 2 days, and transferred to a DIK-710A Seed Pack (Daiki Rika Co., Ltd.) with B\&D nitrogen-free solution. On the following day (the third day of germination), seeds were inoculated with $2 \times 10^{7}$ cells $/ \mathrm{mL}$ bacterial cultures (3 replicates of seeds for each sample). Then, three days after inoculation, the soybean $n f r 1$ mutant roots were immediately frozen in liquid nitrogen and then ground into a fine powder; $100 \mathrm{mg}$ of the powder was measured and used for total RNA extraction using RNeasy Plant Mini Kit (Qiagen) and treated with DNase I (Qiagen) according to the manufacturer's instructions. RNA quality and concentration were evaluated using a NanoDrop 2000/200c (Thermo Fisher). The measured A260/280 and A260/230 ratios were 2.1 and 2.1 to 2.4, respectively, in the case of all samples. The cDNA library was constructed according to the True Stranded mRNA Sample Preparation Guide, Part \#15031047 Rev. E Protocol by using an LT Sample Prep Kit (Illumina).

Read trimming and mapping to the reference genome. The 'raw reads' obtained were filtered to remove lowquality and contaminant reads, including adaptor sequences, DNA, or PCR duplicates, which generated trimmed reads. The trimmed reads were mapped to the reference genome Glycine_max_v2.1 by using HISAT2 version 2.1.0 and Bowtie2 2.3.4.1 software (https://ccb.jhu.edu/software/hisat2/index.shtml). The aligned reads were transcribed using StringTie version 1.3.4d (https://ccb.jhu.edu/software/stringtie/), and expression profiles were normalized to FPKM (fragments per kilobase of transcript per million mapped reads) or RPKM (reads per kilobase of transcript per million mapped reads) values.

Screening of DEGs and enrichment analysis. Bel2-5-dependent DEGs were defined using the following criteria: $p<0.05$; fold change $\geq 1.5$ as upregulated, and fold change $\leq 0.05$ as downregulated. The classified genes were examined for enrichment and annotation using Blast2GO software ${ }^{69}$ and QuickGO (https://www.ebi.ac.uk/ QuickGO/).

qRT-PCR analysis for selected soybean DEGs. For qRT-PCR of selected soybean DEGs, including three defenseand symbiosis-related genes were measured using the same protocol with Bel2-5 expression quantification described previously ${ }^{16}$. The primer set used in qRT-PCR are listed in Supplementary Table 4. Transcript levels of selected soybean DEGs was normalized to the expression of its housekeeping gene SUBI- $2^{70}$ measured in the same samples.

Microscopy. For microscopy analysis, nodules were sectioned using a microtome (VT1000s; Leica Biosystem, Germany) and examined using an SZX9 Microscope (Olympus, Japan).

Bioinformatics and statistical analysis. NLSs were predicted using NLS Mapper (http://nls-mappe r.iab.keio.ac.jp/cgi-bin/NLS_Mapper_form.cgi). ULPs domain were inspected using NCBI Conserved Domain Search (https://www.ncbi.nlm.nih.gov/Structure/cdd/wrpsb.cgi). The Bel2-5 repeat domain was analysed using Tandem Repeats Finder ${ }^{71}$ (https://tandem.bu.edu/trf/trf.html). Homology searches were performed using BLASTP from the National Center for Biotechnology Information (NCBI) (https://blast.ncbi.nlm.nih.gov/) and MEROPS from the Peptidase Database (https://www.ebi.ac.uk/merops/submit_searches.shtml). Bel2-5 and its homologues were aligned using the MUSCLE or CLUSTALW algorithm. The phylogenetic tree was constructed by using MEGA 7 software with the neighbour-joining method and 1000 bootstrap replicates ${ }^{72}$. Data were analysed using Student's $t$-test and Tukey's Honestly Significant Difference (HSD) test, performed using IBM SPSS Statistics 22.0 software.

\section{Data availability}

The RNA-seq data discussed in this publication have been deposited in DDBJ's Sequence Read Archive (DRA) (https://www.ddbj.nig.ac.jp/dra/index-e.html) repository and are accessible through GEO Series Accession Number DRA010122.

Received: 18 September 2020; Accepted: 6 January 2021

Published online: 21 January 2021

\section{References}

1. Oldroyd, G. E. D., Murray, J. D., Poole, P. S. \& Downie, J. A. The rules of engagement in the legume-rhizobial symbiosis. Annu. Rev. Genet. 45, 119-144 (2011).

2. Bohlool, B. B., Ladha, J. K., Garrity, D. P. \& George, T. Biological nitrogen fixation for sustainable agriculture: a perspective. Plant Soil 141, 1-11 (1992). 
3. Burris, R. H. \& Roberts, G. P. Biological nitrogen. Annu. Rev. Nutr. 13, 317-335 (1993).

4. Oldroyd, G. E. D. Speak, friend, and enter: signalling systems that promote beneficial symbiotic associations in plants. Nat. Rev. Microbiol. 11, 252-263 (2013).

5. Radutoiu, S. et al. Plant recognition of symbiotic bacteria requires two LysM receptor-like kinases. Nature 425, 585-592 (2003).

6. Madsen, E. B. et al. A receptor kinase gene of the LysM type is involved in legume perception of rhizobial signals. Chemtracts 17, 508-514 (2004).

7. Kawaharada, Y. et al. Receptor-mediated exopolysaccharide perception controls bacterial infection. Nature 523, 308-312 (2015).

8. Simsek, S., Ojanen-Reuhs, T., Stephens, S. B. \& Reuhs, B. L. Strain-ecotype specificity in Sinorhizobium meliloti-Medicago truncatula symbiosis is correlated to succinoglycan oligosaccharide structure. J. Bacteriol. 189, 7733-7740 (2007).

9. Becker, A., Fraysse, N. \& Sharypova, L. Recent advances in studies on structure and symbiosis-related function of rhizobial K-antigens and lipopolysaccharides. Mol. Plant Microbe Interact. 18, 899-905 (2005).

10. Miwa, H. \& Okazaki, S. How effectors promote beneficial interactions. Curr. Opin. Plant Biol. 38, 148-154 (2017).

11. Staehelin, C. \& Krishnan, H. B. Nodulation outer proteins: double-edged swords of symbiotic rhizobia. Biochem. J. 470, 263-274 (2015).

12. Xin, D. W. et al. Functional analysis of NopM, a novel E3 ubiquitin ligase (NEL) domain effector of Rhizobium sp. strain NGR234. PLoS Pathog. 8, e1002707 (2012).

13. Zhang, L., Chen, X. J., Lu, H. B., Xie, Z. P. \& Staehelin, C. Functional analysis of the type 3 effector nodulation outer protein L (NopL) from Rhizobium sp. NGR234: symbiotic effects, phosphorylation, and interference with mitogen-activated protein kinase signaling. J. Biol. Chem. 286, 32178-32187 (2011).

14. Nguyen, H. P., Ratu, S. T. N., Yasuda, M., Göttfert, M. \& Okazaki, S. InnB, a novel type III effector of Bradyrhizobium elkanii USDA61, controls symbiosis with Vigna species. Front. Microbiol. 9, 3155 (2018).

15. Faruque, O. M. et al. Identification of Bradyrhizobium elkanii genes involved in incompatibility with soybean plants carrying the Rj4 allele. Appl. Environ. Microbiol. 81, 6710-6717 (2015).

16. Yasuda, M. et al. Effector-triggered immunity determines host genotype-specific incompatibility in legume-rhizobium symbiosis. Plant Cell Physiol. 57, 1791-1800 (2016).

17. Okazaki, S., Kaneko, T., Sato, S. \& Saeki, K. Hijacking of leguminous nodulation signaling by the rhizobial type III secretion system. Proc. Natl. Acad. Sci. 110, 17131-17136 (2013).

18. Okazaki, S. et al. Rhizobium-legume symbiosis in the absence of Nod factors: two possible scenarios with or without the T3SS. ISME J. 10, 64-74 (2016).

19. Teulet, A. et al. The rhizobial type III effector ErnA confers the ability to form nodules in legumes. Proc. Natl. Acad. Sci. USA 116, 21758-21768 (2019).

20. Krause, A., Doerfel, A. \& Göttfert, M. Mutational and transcriptional analysis of the type III secretion system of Bradyrhizobium japonicum. Mol. Plant Microbe Interact. 15, 1228-1235 (2002).

21. Wassem, R. et al. TtsI regulates symbiotic genes in Rhizobium species NGR234 by binding to tts boxes. Mol. Microbiol. 68, 736-748 (2008)

22. Okazaki, S., Zehner, S., Hempel, J., Lang, K. \& Göttfert, M. Genetic organization and functional analysis of the type III secretion system of Bradyrhizobium elkanii. FEMS Microbiol. Lett. 295, 88-95 (2009).

23. Wenzel, M., Friedrich, L., Göttfert, M. \& Zehner, S. The type III-secreted protein NOpEl affects symbiosis and exhibits a calciumdependent autocleavage activity. Mol. Plant Microbe Interact. 23, 124-129 (2010).

24. Skorpil, P. et al. NopP, a phosphorylated effector of Rhizobium sp. strain NGR234, is a major determinant of nodulation of the tropical legumes Flemingia congesta and Tephrosia vogelii. Mol. Microbiol. 57, 1304-1317 (2005).

25. López-Baena, F. J. et al. The absence of nops secretion in Sinorhizobium fredii HH103 increases GmPR1 expression in williams soybean. Mol. Plant Microbe Interact. 22, 1445-1454 (2009).

26. Guttman, D. S. et al. A functional screen for the type III (Hrp) secretome of the plant pathogen Pseudomonas syringae. Science 295, 1722-1726 (2002).

27. Petnicki-Ocwieja, T. et al. Genomewide identification of proteins secreted by the Hrp type III protein secretion system of Pseudomonas syringae pv. tomato DC3000. Proc. Natl. Acad. Sci. USA 99, 7652-7657 (2002).

28. Kosugi, S., Hasebe, M., Tomita, M. \& Yanagawa, H. Systematic identification of cell cycle-dependent yeast nucleocytoplasmic shuttling proteins by prediction of composite motifs. Proc. Natl. Acad. Sci. USA 106, 10171-10176 (2009).

29. Hotson, A., Chosed, R., Shu, H., Orth, K. \& Mudgett, M. B. Xanthomonas type III effector XopD targets SUMO-conjugated proteins in planta. Mol. Microbiol. 50, 377-389 (2003).

30. Li, S. J. \& Hochstrasser, M. A new protease required for cell-cycle progression in yeast. Nature 398, 246-251 (1999).

31. Xiang, Q. W. et al. NopD of Bradyrhizobium sp. XS1150 possesses SUMO protease activity. Front. Microbiol. 11, 1-12 (2020).

32. Hickey, C. M., Wilson, N. R. \& Hochstrasser, M. Function and regulation of SUMO proteases. Nat. Rev. Mol. Cell Biol. 13, 755-766 (2012).

33. Ohta, M., Matsui, K., Hiratsu, K., Shinshi, H. \& Ohme-takagi, M. Repression domains of class II ERF transcriptional repressors share an essential motif for active repression. Plant Cell 13, 1959-1968 (2001).

34. Kazan, K. Negative regulation of defence and stress genes by EAR-motif-containing repressors. Trends Plant Sci. 11, 109-112 (2006).

35. Kim, J. G. et al. XopD SUMO protease affects host transcription, promotes pathogen growth, and delays symptom development in Xanthomonas-infected tomato leaves. Plant Cell 20, 1915-1929 (2008).

36. Kim, J. G., Taylor, K. W. \& Mudgett, M. B. Comparative analysis of the XopD type III secretion (T3S) effector family in plant pathogenic bacteria. Mol. Plant Pathol. 12, 715-730 (2011).

37. Dean, P. Functional domains and motifs of bacterial type III effector proteins and their roles in infection. FEMS Microbiol. Rev. 35, 1100-1125 (2011).

38. Deakin, W. J., Marie, C., Saad, M. M., Krishnan, H. B. \& Broughton, W. J. NopA is associated with cell surface appendages produced by the type III secretion system of Rhizobium sp. strain NGR234. Mol. Plant Microbe Interact. 18, 499-507 (2005).

39. Saad, M. M., Staehelin, C., Broughton, W. J. \& Deakin, W. J. Protein-protein interactions within type III secretion system-dependent pili of Rhizobium sp. strain NGR234. J. Bacteriol. 190, 750-754 (2008).

40. Sory, M.-P. \& Cornelis, G. R. Translocation of a hybrid YopE-adenylate cyclase from Yersinia enterocolitica into HeLa cells. Mol. Microbiol. 14, 583-594 (1994).

41. Hotson, A. \& Mudgett, M. B. Cysteine proteases in phytopathogenic bacteria: identification of plant targets and activation of innate immunity. Curr. Opin. Plant Biol. 7, 384-390 (2004).

42. El-Showk, S., Ruonala, R. \& Helariutta, Y. Crossing paths: cytokinin signalling and crosstalk. Development 140, 1373-1383 (2013).

43. Nizampatnam, N. R., Schreier, S. J., Damodaran, S., Adhikari, S. \& Subramanian, S. MicroRNA160 dictates stage-specific auxin and cytokinin sensitivities and directs soybean nodule development. Plant J. 84, 140-153 (2015).

44. Mens, C., Li, D., Haaima, L. E., Gresshoff, P. M. \& Ferguson, B. J. Local and systemic effect of cytokinins on soybean nodulation and regulation of their Isopentenyl Transferase (IPT) biosynthesis genes following Rhizobia inoculation. Front. Plant Sci. 9, 1-14 (2018).

45. Dao, T. T. H., Linthorst, H. J. M. \& Verpoorte, R. Chalcone synthase and its functions in plant resistance. Phytochem. Rev. 10, 397-412 (2011). 
46. Kachroo, P., Shanklin, J., Shah, J., Whittle, E. J. \& Klessig, D. F. A fatty acid desaturase modulates the activation of defense signaling pathways in plants. Proc. Natl. Acad. Sci. USA 98, 9448-9453 (2001).

47. Tang, D., Ade, J., Frye, C. A. \& Innes, R. W. Regulation of plant defense responses in Arabidopsis by EDR2, a PH and START domain-containing protein. Plant J. 44, 245-257 (2005).

48. Kim, M. C. et al. Mlo, a modulator of plant defense and cell death, is a novel calmodulin-binding protein. Isolation and characterization of a rice Mlo homologue. J. Biol. Chem. 277, 19304-19314 (2002).

49. Kim, M. C. et al. Calmodulin interacts with MLO protein to regulate defence against mildew in barley. Nature 416, 447-450 (2002).

50. Shen, Q. et al. Genome-scale identification of MLO domain-containing genes in soybean (Glycine max L. Merr.). Genes Genet. Syst. 87, 89-98 (2012).

51. Guinel, F. C. Ethylene, a hormone at the center-stage of nodulation. Front. Plant Sci. 6, 1121 (2015).

52. Andriankaja, A. et al. AP2-ERF transcription factors mediate nod factor-dependent Mt ENOD11 activation in root hairs via a novel cis-regulatory motif. Plant Cell 19, 2866-2885 (2007).

53. Liang, Y. et al. Nonlegumes respond to rhizobial nod factors by suppressing the innate immune response. Science 341, 1384-1387 (2013).

54. Marie, C. et al. Characterization of Nops, nodulation outer proteins, secreted via the type III secretion system of NGR234. Mol. Plant Microbe Interact. 16, 743-751 (2003).

55. Ge, Y. Y. et al. The type 3 effector NopL of Sinorhizobium sp. strain NGR234 is a mitogen-activated protein kinase substrate. J. Exp. Bot. 67, 2483-2494 (2016).

56. Noël, L., Thieme, F., Nennstiel, D. \& Bonas, U. Two novel type III-secreted proteins of Xanthomonas campestris pv. vesicatoria are encoded within the hrp pathogenicity Island. J. Bacteriol. 184, 1340-1348 (2002).

57. Gill, G. SUMO and ubiquitin in the nucleus: different functions, similar mechanisms?. Genes Dev. 18, 2046-2059 (2004).

58. Li, Y. et al. Organization and regulation of soybean SUMOylation system under abiotic stress conditions. Front. Plant Sci. 8, 1-14 (2017).

59. Kim, J. G., Stork, W. \& Mudgett, M. B. Xanthomonas type III effector XopD desumoylates tomato transcription factor SlERF4 to suppress ethylene responses and promote pathogen growth. Cell Host Microbe 13, 143-154 (2013).

60. Murray, J. D. et al. A cytokinin perception mutant colonized by Rhizobium in the absence of nodule organogenesis. Science 315, 101-104 (2007).

61. Reid, D. et al. Cytokinin biosynthesis promotes cortical cell responses during nodule development. Plant Physiol. 175, 361-375 (2017).

62. Sadowsky, M. J., Tully, R. E., Cregan, P. B. \& Keyser, H. H. Genetic Diversity in Bradyrhizobium japonicum serogroup 123 and its relation to genotype-specific nodulation of soybean. Appl. Environ. Microbiol. 53, 2624-2630 (1987).

63. Green, M. \& Sambrook, J. Molecular Cloning a Laboratory Manual 33 (Cold Spring Harbor Laboratory Press, Cold Spring Harbor, 2012).

64. Regensburger, B. \& Hennecke, H. RNA polymerase from Rhizobium japonicum. Arch. Microbiol. 135, 103-109 (1983).

65. Francisco, P. B. \& Akao, S. Autoregulation and nitrate inhibition of nodule formation in soybean cv. enrei and its nodulation mutants. J. Exp. Bot. 44, 547-553 (1993).

66. Broughton, W. J. \& Dilworth, M. J. Control of leghaemoglobin synthesis in snake beans. Biochem. J. 125, 1075-1080 (2015).

67. Wen, S., Chen, X., Xu, F. \& Sun, H. Validation of reference genes for real-time quantitative PCR (qPCR) analysis of avibacterium paragallinarum. PLoS ONE 11, 1-14 (2016).

68. Süß, C. et al. Identification of genistein-inducible and type III-secreted proteins of Bradyrhizobium japonicum. J. Biotechnol. 126, 69-77 (2006).

69. Götz, S. et al. High-throughput functional annotation and data mining with the Blast2GO suite. Nucleic Acids Res. 36, 3420-3435 (2008).

70. Brechenmacher, L. et al. Transcription profiling of soybean nodulation by Bradyrhizobium japonicum. Mol. Plant Microbe Interact. 21, 631-645 (2008).

71. Benson, G. Tandem repeats finder: a program to analyze DNA sequences. Nucleic Acids Res. 27, 573-580 (1999).

72. Kumar, S., Stecher, G. \& Tamura, K. MEGA7: molecular evolutionary genetics analysis version 7.0 for bigger datasets. Mol. Biol. Evol. 33, 1870-1874 (2016).

\section{Acknowledgements}

This study was supported by JSPS Kakenhi (19H02860 and 19K22303) and the Agence Nationale de la Recherche, Grant "SymEffectors", No. ANR-16-CE20-0013.

\section{Author contributions}

S.T.N.R., E.G. and S.O. designed the experiments. S.T.N.R., A.T., H.M., S.M., H.P.N., M.Y., S.S., T.K., M.H. performed experiments and analysed the data. S.T.N.R., E.G. and S.O. wrote the paper.

\section{Competing interests}

The authors declare no competing interests.

\section{Additional information}

Supplementary Information The online version contains supplementary material availlable at https://doi. org/10.1038/s41598-021-81598-6.

Correspondence and requests for materials should be addressed to S.O.

Reprints and permissions information is available at www.nature.com/reprints.

Publisher's note Springer Nature remains neutral with regard to jurisdictional claims in published maps and institutional affiliations. 
(c) (i) Open Access This article is licensed under a Creative Commons Attribution 4.0 International cc) License, which permits use, sharing, adaptation, distribution and reproduction in any medium or format, as long as you give appropriate credit to the original author(s) and the source, provide a link to the Creative Commons licence, and indicate if changes were made. The images or other third party material in this article are included in the article's Creative Commons licence, unless indicated otherwise in a credit line to the material. If material is not included in the article's Creative Commons licence and your intended use is not permitted by statutory regulation or exceeds the permitted use, you will need to obtain permission directly from the copyright holder. To view a copy of this licence, visit http://creativecommons.org/licenses/by/4.0/.

(C) The Author(s) 2021 